The Egyptian Journal of Hospital Medicine (January 2019) Vol. 74 (3), Page 511-516

\title{
Dosimetric Study Comparing Three-Dimensional Conformal Radiotherapy to Intensity Modulated Radiotherapy in Management of Rectal Carcinoma. Retrospective study
}

\author{
Mahmoud M. Shaban, Mohamed ElSayed Mansy, Khaled M. El Shahat \\ Clinical Oncology Department, Faculty of Medicine, Al Azhar University, Cairo-Egypt \\ Corresponding author: Khaled M. El Shahat, email:khelshahat@yahoo.com
}

\begin{abstract}
Background: the External radiotherapy (EBRT) is the common treatment selection in patients with locally advanced rectum carcinoma. Three-dimensional conformal radiotherapy (3DCRT) and intensity modulated radiotherapy (IMRT) are most techniques used in radiotherapy. However, which one is superior is all controversial. Patients and Methods: thirty-two patients of RC treated with IMRT were selected for current retrospective study. 3DCRT plans were also plan for all the patients. 3DCRT plans in compared to IMRT plans were on the basis of planning target volume (PTV) coverage, dose to normal organs, homogeneity index (HI) and conformity index (CI). Results and Discussions: in both the planning techniques $98 \%$ of PTV was covered with more than $96 \%$ of prescribed dose. D 95\% 15, D 35 and $\mathrm{D}$ (Dose to $15 \%, 35 \%$ and $50 \%$ volume respectively) for bladder was reduced by $2.09 \%, 14.623 \%$ and $32.57 \%$ in IMRT compared to 3DCRT. V (Volume received 45 Gy also volume received 50.4Gy) and in case of bowel doses were found to be better in IMRT than to 3DCRT. There is significant difference found between doses to both femoral heads in IMRT and 3DCRT. The CI 95\% 45 in IMRT plans was found much better than that in 3DCRT whereas HI in both the techniques were found almost same. Conclusion: IMRT in better significantly in reduction the irradiated of OAR and improved dose conformity in the PTV compared to that by 3DCRT. So, it can be concluded that IMRT should be chosen as best technique for the radiotherapy of Rectum carcinoma
\end{abstract}

Keywords: IMR, rectal Carcinoma, treatment planning, dosimetric comparison, 3DCRT

\section{Introduction:}

For many years surgery has been regarded as the main treatment for rectal carcinoma. Total meso-rectal excision (TME) is best selection over Abdominoperineal excision (APR) due to its better overall good survival rate and a lower of tumor recurrence ${ }^{(\mathbf{1})}$.Colorectal carcinoma has been considered the second most common cause of cancer in women $(9.2 \%$ of diagnoses) and the third most common in men $(10.0 \%)$ in the year 2012, worldwide more than one million people are diagnosed annually of colorectal carcinoma resulting in about 715,000 deaths as of 2010 up from 490,000 in 1990 ${ }^{(2,3)}$.The development in the treatment planning, the procedure of radiotherapy by a ThreeDimensional Conformal RT (3D-CRT), technique for treating rectal cancer and advance techniques like IMRT as advance modalities for of radiotherapy delivery continues to be utilized by various cancer centers in many countries, such as Egypt due to upgrading in the infrastructure of availability of Machines equipment by advance techniques like IMRT in many center in Egypt and staff have good training for new advances of radiotherapy ${ }^{(4)}$. In addition, the good results for treatment approaches, for advance techniques using in radiotherapy for reduction dose to organs at risk like for tumor site head and neck, prostate and etc. there are very small number of studies that aim to evaluate the advance techniques start from 3 DCRT and IMRT in consider to target volume coverage by prescribed dose and dose to organs at risk (OAR) ${ }^{(5,6)}$. The current study for compare the dosimetric tools of 3 DCRT Varus IMRT in terms of target coverage and doses to OAR in the control of rectal carcinoma ${ }^{(7-8)}$.

\section{Patients and Methods}

This retrospective observational dosimetric study had been carried out at the clinical oncology department at Al-Hussein university hospital. Thirty patients with pathological 
evidence confirmed rectum cancer and radiological evidence of being non-metastatic who received local radiation therapy treated with 3DCRT between the year 2012 and the year 2017 shall be collected from department archive and the clinico-epidemiological data will be retrieved as well as the radiation therapy details stored in the planning computer system will be retrieved also. As shown in the fig.1. the sex and age. distribution for patients were included current study. The study was approved by the Ethics Board of Al-Azhar University.
Inclusion criteria:

- $\quad$ Male and female Patients between 18 and 75 years old.

- Histo-pathologically proven to have rectal carcinoma.

- $\quad$ Stage I, II and III rectal carcinoma patients.

- Patients treated by either threedimensional conformal radiotherapy (3DCRT) or intensity modulated radiotherapy (IMRT) in either neoadjuvant or adjuvant settings.

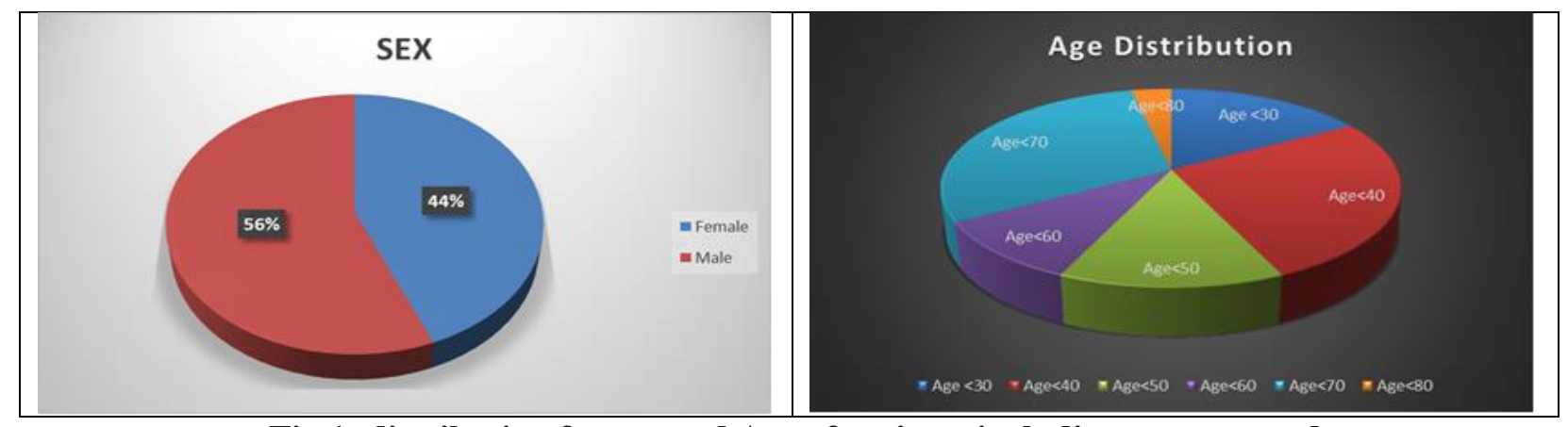

Fig 1: distribution for sex and Age of patients including current study

\section{Radiotherapy technique:}

The whole study group going to receive preoperative pelvic radiation therapy concurrent with chemotherapy. Localization, immobilization, and simulation: - During simulation and treatment, the patient is prone with a full bladder to decrease the small intestinal dose, rectal and vaginal markers are used during simulation. The laser localizer system is used to put a 3 radio opaque marks for position reproducibility, these marks are used as references for repositioning of the patient for treatment delivery ${ }^{(9)}$. The patient then was scanned by CT scan machine from the ischial tuberosities up to the lower border of L5 with cuts interval $5 \mathrm{~mm}$ (GE Lightspeed GE Healthcare). In order to make a dosimetric comparison, IMRT treatment plans were generated for the same thirty patients. Acute effects and late effects in addition probability for toxicity of treatment were explained by simple way to all patients, the study was accepted by the university ethics committee. Varian - eclipse planning system version 15.7 was used as an algorithm for planning. Body was contoured generated by automatic method by treatment planning system and surrounding critical structures were delineations manually. Clinical Target Volume (CTV). Planning Target Volume (PTV) was created by a $10 \mathrm{~mm}$ expansion around CTV in all surrounding. Target and critical organ delineations were performed by the same radiation oncologist. To perform Dosimetric comparison for Three-Dimensional Conformal Radiotherapy (3DCRT) versus Intensity Modulated Radiotherapy (IMRT) Rectal carcinoma in terms of: Organs at risk (OAR) dose. Conformity index (CI), Planning target volume (PTV) coverage, Homogeneity index (HI), Time for delivery and Dose monitor units (MU) Low dose in body (Integral Dose) (10).

\section{Results and Discussions: -}

PTV 50.4 GY COVERAGE as shown in fig.2

The 95\% coverage of 3DCRT PTV 50.4Gy plan showed mean dose of 48.32 Gy $(\mathrm{SD} \pm 0.52)$, median dose of 47.88 Gy, minimum dose recorded 47.8 Gy $(94.8 \%)$ and maximum dose recorded $49.31 \mathrm{GY}(97.8 \%)$. Meanwhile $95 \%$ of IMRT 50.4 Gy plans showed man dose of 48.27 Gy (SD \pm 043$)$, median dose of $48.12 \mathrm{~Gy}$, 
minimum value recorded $47.8(94.8 \%)$ and maximum value recorded 49.35 Gy $(98.2 \%)$. Comparing these data together showed no significant difference between 3DCRT and IMRT plans in PTV 50.4 coverage. $(\mathrm{p}=$ 0.72764).

\section{PTV 45 GY COVERAGE}

The $95 \%$ coverage of 3DCRT PTV 45Gy plan showed mean dose of 44.42 Gy $(\mathrm{SD} \pm 0.92)$, median dose of $44.30 \mathrm{~Gy}$, minimum dose recorded 43.07 Gy $(95.7 \%)$ and maximum dose recorded $45.97 \mathrm{GY}(102 \%)$. Meanwhile $95 \%$ of IMRT 50.4 Gy plans showed man dose of 44.50 Gy (SD \pm 043$)$, median dose of $45.91 \mathrm{~Gy}$, minimum value recorded $42.96(95.4 \%)$ and maximum value recorded $45.97 \mathrm{~Gy}(102 \%)$. comparing these data together showing no significant difference between 3DCRT and IMRT plans in PTV 45 Gy coverage. ( $\mathrm{p}=$ 0.76684 ). These results data are comparable with similar publish paper ${ }^{(12)}$.

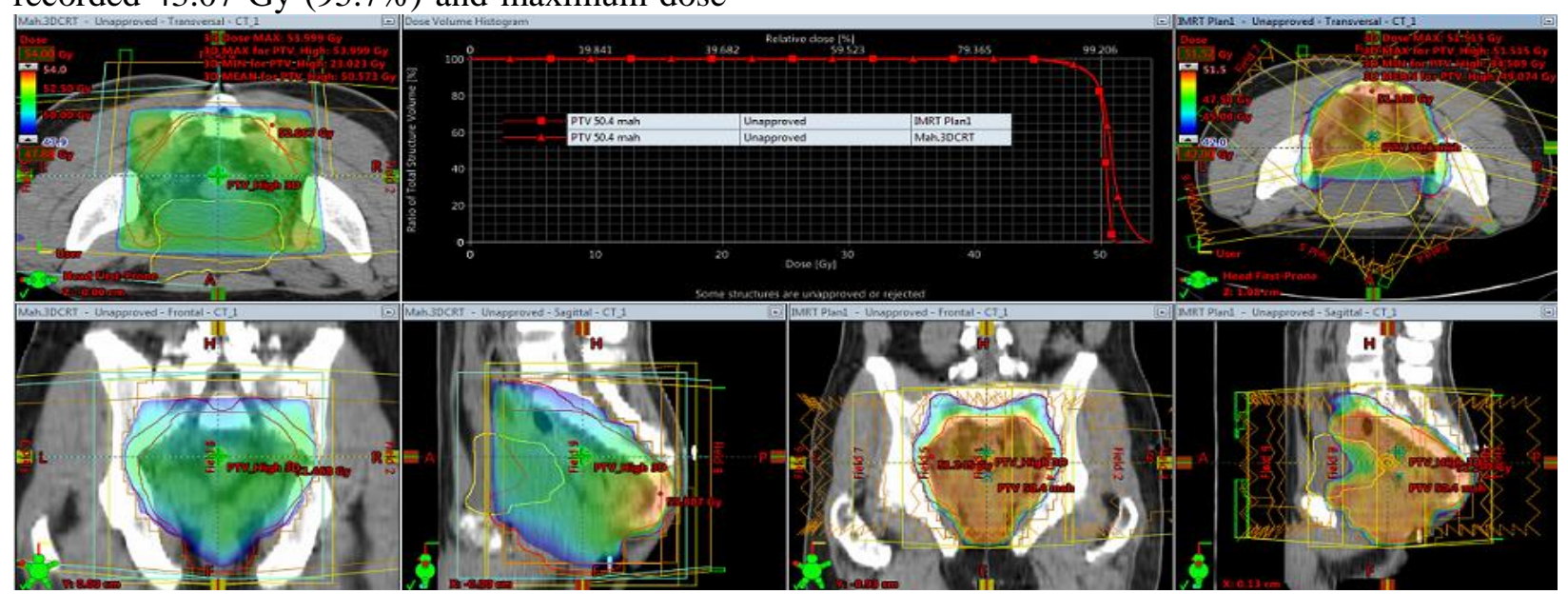

Fig 2: the different views for comparison between 3DCRT and IMRT plan for target volume coverage

\section{Conformity Index (CI).}

Conformity index of 3DCRT showing mean value of $0.69(\mathrm{SD} \pm 0.056)$ median value 0.7 , minimum value 0.58 and maximum value 0.78 mean while conformity index of IMRT plans showing mean value of 0.87 (SD \pm 0.036 ), median value of 0.85 , minimum value of 0.80 and maximum value of $0.930 . \quad(p=$ 2.29369E-21). Comparing these results together showed significant difference in favor of IMRT technique.

\section{Homogeneity index (HI):}

Homogeneity index of 3DCRT plans showed mean value of $0.109(\mathrm{SD} \pm 0.023$ ) median value 0.1 minimum value 0.07 and maximum value 0.17 mean while conformity index of IMRT plans showing mean value of 0.132 (SD \pm 0.13 ), median value of 0.13 , minimum value of 0.10 and maximum value of 0.18. Comparing these results together showed a significant difference in favor of IMRT technique. These results data were comparable with similar study performed by Ngan $\boldsymbol{e t} \boldsymbol{a l}$. evaluated target dose delivery, and the same plan evaluation parameters. Target dose distribution was more inhomogeneous following IMRT planning while lower conformality index for 3DCRT plans. The mean and maximum values for OAR were radically decreased in the IMRT plans $(\mathrm{p}<0.05)$. They concluded that IMRT planning was superior for target conformality and reduced irradiation of the organs at risks at the cost of increased target heterogeneity ${ }^{(13)}$.

\section{Bladder comparison between IMRT and 3DCRT}

The maximum dose of the urinary bladder in 3DCRT plans showing mean value of 49.26 Gy(SD \pm 0.669$)$, median value of $49.15 \mathrm{~Gy}$,minimum value of $47.43 \mathrm{GY}$ and maximum value of $49.90 \mathrm{~Gy}$, meanwhile the urinary bladder maximum dose in IMRT plans showing mean value of $44.80 \mathrm{~Gy}(\mathrm{SD} \pm 2.915)$, median value of $45.45 \mathrm{~Gy}$, minimum value of 37.14Gyand maximum value of $48.91 \mathrm{~Gy}$, comparing these data together showing significant difference in favor of IMRT technique.as shown in the figure $3^{(13)}$. 


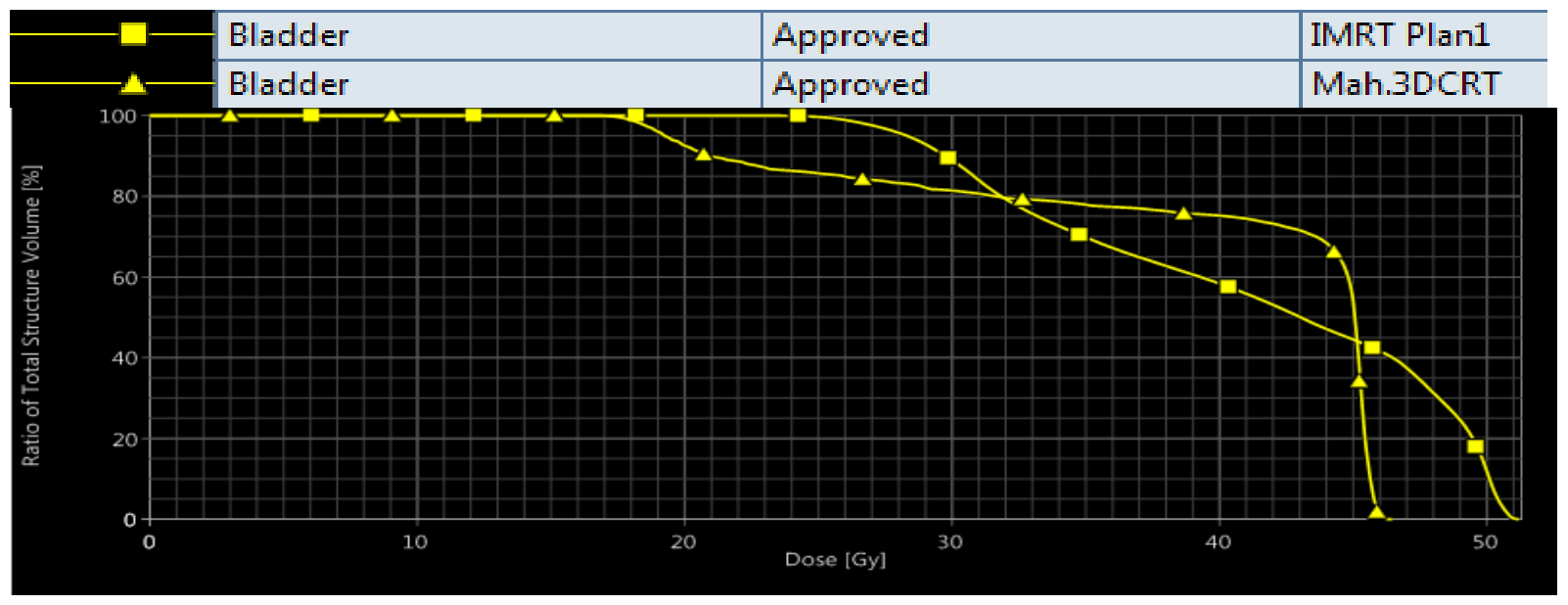

Fig 3: bladder dose comparison for both plans.

\section{Mean Dose to the Both Femoral heads}

Right femur head and neck mean dose in 3DCRT plans showed mean value of $25.93 \mathrm{~Gy}$ ( $\mathrm{SD} \pm 3.71$ ), median value of $26.16 \mathrm{~Gy}$, minimum value of $12.54 \mathrm{~Gy}$ and maximum value of 36.34 Gy, meanwhile mean dose IMRT plans showing mean value of 11.95 Gy ( $\mathrm{SD} \pm 3.42$ ), median value of $10.81 \mathrm{~Gy}$, minimum value of $4.80 \mathrm{~Gy}$ and maximum value of $17.39 \mathrm{~Gy}$, comparing these data together showed significant difference in favor of IMRT technique. Left femur head and neck mean dose in 3DCRT plans showing

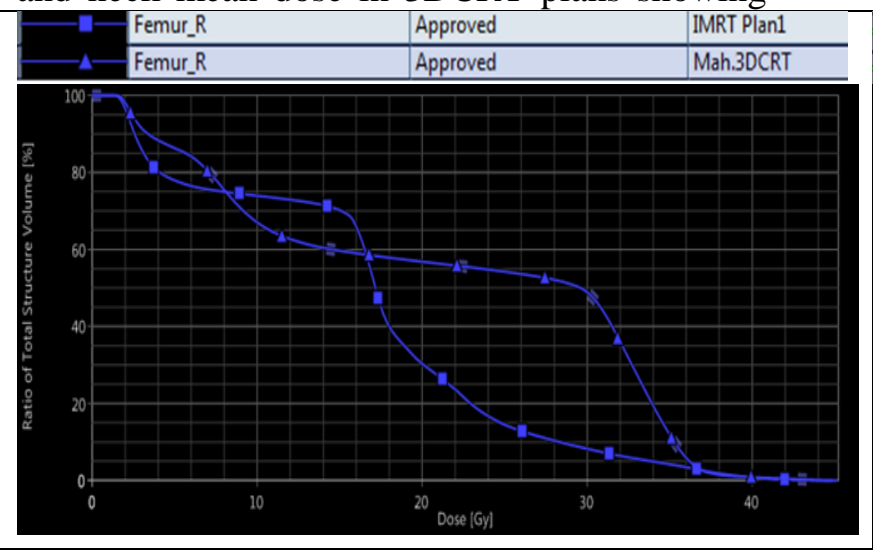

mean value of 26.32 Gy ( $\mathrm{SD} \pm 4.86$ ), median value of $22.63 \mathrm{~Gy}$, minimum value of $15.39 \mathrm{~Gy}$ and maximum value of $36.34 \mathrm{~Gy}$, meanwhile mean dose IMRT plans showed mean value of 11.95 Gy ( $\mathrm{SD} \pm 3.27$ ), median value of $11.95 \mathrm{~Gy}$, minimum value of $5.79 \mathrm{~Gy}$ and maximum value of 16.81, comparing these data together showing significant difference in favor of IMRT technique. As illustrated in the fig.4. These results data are comparable with published paper (14)

Fig 4: both femoral heads mean dose for both plans

\section{BONE MARROW MEAN DOSE:}

Bone marrow mean dose in 3DCRT plans showing mean value of 30.88Gy (SD \pm 2.75 ), median value of $31.30 \mathrm{~Gy}$, minimum value of 27.03 Gy and maximum value of $35.34 \mathrm{~Gy}$, meanwhile mean dose IMRT plans showing mean value of $26.08 \mathrm{~Gy}(\mathrm{SD}$

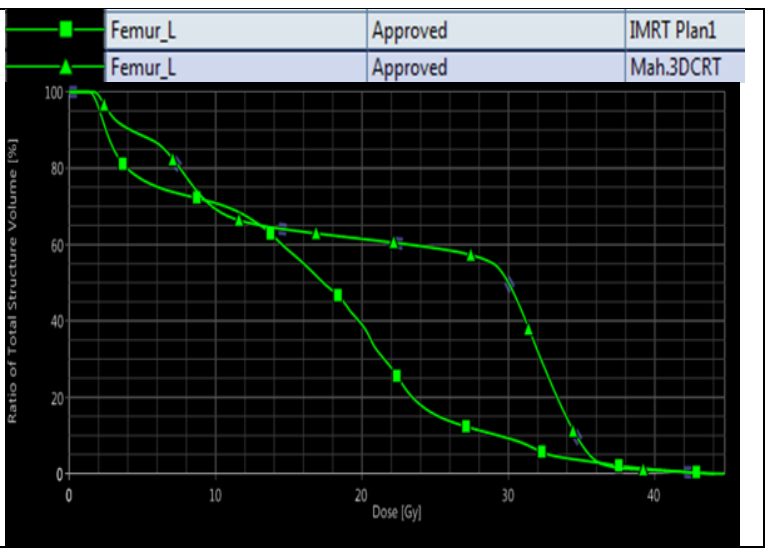

\pm 2.731 ), median value of $26.45 \mathrm{~Gy}$, minimum value of $18.89 \mathrm{~Gy}$ and maximum value of 29.91Gy, comparing these data together showing significant difference in favor of IMRT technique. $(\mathrm{p}=1.08849 \mathrm{E}-8)$ as shown in figure 5. 


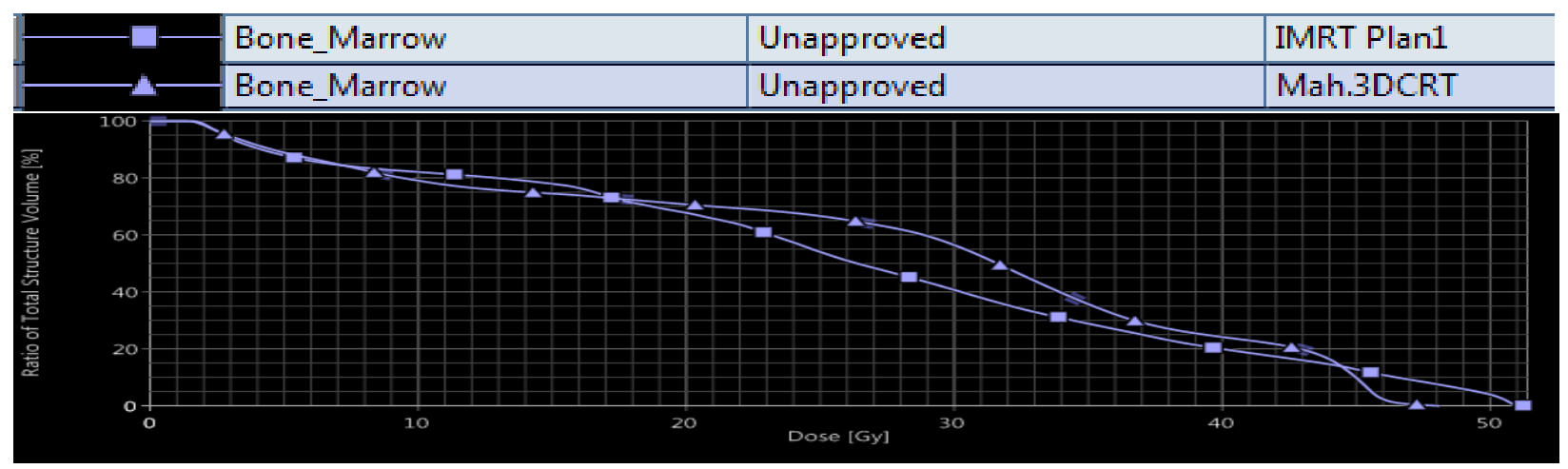

Fig. 5: bone marrow dose for both plans

\section{BOWEL MEAN DOSE:}

Bowel mean dose in 3DCRT plans showed mean value of $22.49 \mathrm{GY}$ ( $\mathrm{SD} \pm 5.33$ ), median value of $22.92 \mathrm{~Gy}$ minimum value of $8.20 \mathrm{~Gy}$ and maximum value of $30.74 \mathrm{GY}$, meanwhile bowel mean dose in IMRT plans showedmean value of 18.78Gy ( $\mathrm{SD} \pm 4.20)$, median value of $19.085 \mathrm{~Gy}$, minimum value of $7.70 \mathrm{GY}$ and maximum value of $28.57 \mathrm{GY}$, comparing these data together showing significant difference in favor of IMRT technique. $(p=0.00464)$ as shown in the Fig. 6 . These results data are comparable with another study that showed that patients who were treated with IMRT had a significant decrease in three or more grades of toxicities $(\mathrm{p}=0.016)$. The occurrence of grade $\geq 3$ diarrhea was $9 \%$ among 3DCRT patients as compared to $3 \%$ of patients receiving IMRT $(\mathrm{p}=0.31)^{(14)}$.

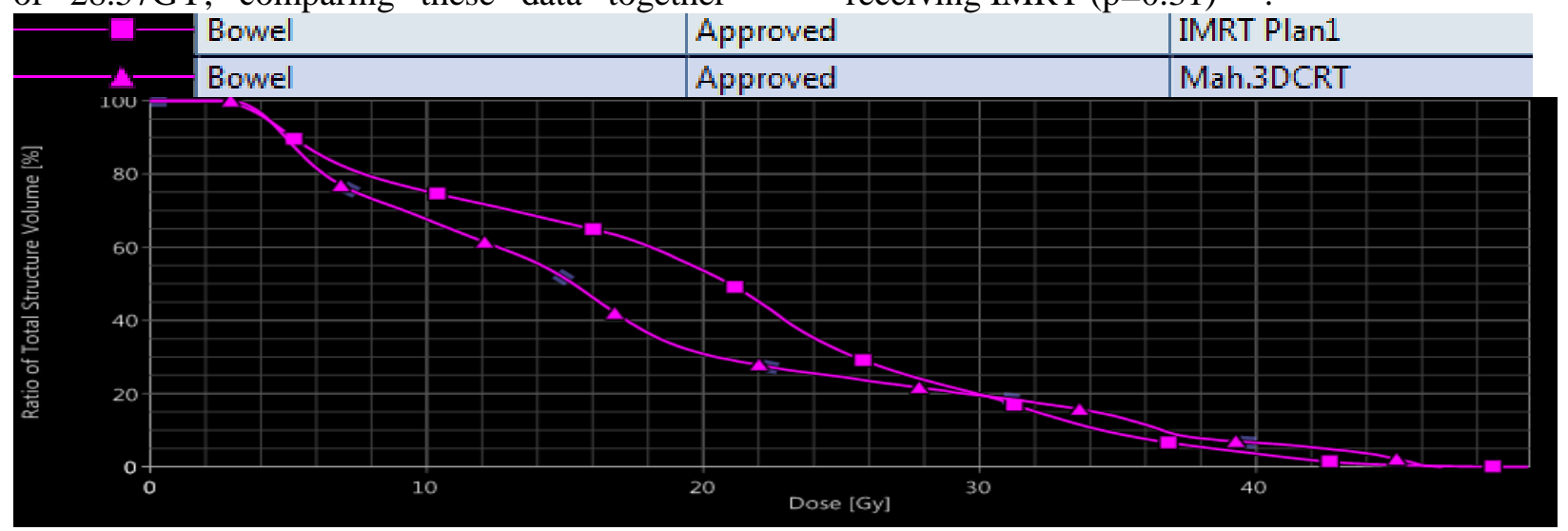

Fig . 6: the comparison mean dose of Bowel in both plans

\section{Duration for treatment:}

According current study the time for IMRT increase more $58 \%$ more than 3DCRT radiotherapy for most case due to number of fields in current Dosimetric study 7 filed.

\section{Monitor unit: -}

According current study the time for IMRT increase more $120 \%$ more than 3DCRT radiotherapy for most case due to number of fields in current Dosimetric study 7 filed, due to many small fields size including each field in IMRT plan.

\section{Conclusion: -}

IMRT is consider as the best selection technique in carcinoma of rectum treatment with less toxicity effects than 3DCRT and delivering better dose for organs at risk. However, according to the current dosimetric outcomes and translates into a significant value and meaningful for clinical benefit and toxicity for surroundings organs.

\section{References:}

1. Ferlay J, Soerjomataram I, Dikshit $\mathbf{R}$ et al. (2015): Cancer incidence and mortality worldwide: sources, methods and major patterns in GLOBOCAN. Int. J. Cancer, 136:359-386.

2. Lozano R, Naghavi M, Foreman $\mathrm{K}$ et al. (2012): Global and regional mortality 
Dosimetric Study Comparing Three-Dimensional Conformal Radiotherapy to Intensity...

from 235 causes of death for 20 age groups in 1990 and 2010: a systematic analysis for the Global Burden of Disease Study .Lancet, 380 (9859): 2095-2100.

3. World Cancer Report (2014): International Agency for Research on Cancer. World Health Organizationpublications.iarc.fr/NonSeries-Publications/World-CancerReports/.

4. Xia P, Fu K, Wong G, Akazawa C and Verhey $L$ J (2000): Comparison of treatment plans involving intensitymodulated radiotherapy for nasopharyngeal carcinoma. International Journal of Radiation Oncology, Biology, Physics, 48: 29-37.

5. Wu Q, Manning M, Schmidt-Ullrich $R$ and Mohan $R$ (2000): The potential for sparing of parotids and escalation of biologically effective dose with intensity-modulated radiation treatments of head and neck cancers: a treatment design study. International Journal of Radiation Oncology, Biology, Physics, 46:195-205.

6. Goyal S, Cohler A, Camporeale J, Narra $V$ and Yue N (2008): Intensitymodulated radiation therapy for orbital lymphoma. Radiation Medicine, 26:573581.

7. Bhatnagar A et al. (2006) Intensity modulated radiation therapy (IMRT) reduces the dose to the contralateral breast when compared to conventional tangential fields for primary breast Iiradiation. Breast Cancer Research and Treatment, 96: 41-46.

8. Zelefsky M et al. (2002): High-dose intensity modulated radiation therapy for prostate cancer: early toxicity and biochemical outcome in 772 patients. International Journal of Radiation Oncology, Biology, Physics, 53:11111116.

9. American Cancer Society (2013): Cancer Facts and Figures. Atlanta, GA. Cancer Society. https://www.cancer.org/.../cancer.../can cer-facts-and-statistics/...cancer-factsand-figur

10. Darlene V, Amr S S, Samuel F, Taylor S, Ahmed H, Mohamed R, Laura S, Ibrahim A (2012) : Age distribution, polyps and rectal cancer in the Egyptian population.World J. Gastroenterol. 18(30): 3997-4003.

11. Jemal A et al. (2011): Global cancer statistics. Cancer J. Clin., 61:69-90.

12. Corner $C$, Khimji $F$ and Tsang $Y$ (2011): Comparison of conventional and three dimensional conformal CT planning techniques for preoperative chemo radiotherapy for locally advanced rectal cancer. $\mathrm{Br}$. J. Radiol.,84(998):173-181.

13. Ngan SY, Burmeister B, Fisher RJ, Solomon M, Goldstein D, Joseph D et al. (2012). Randomized trial of shortcourse radiotherapy versus long-course chemoradiation comparing rates of local recurrence in patients with $\mathrm{T} 3$ rectal cancer: Trans-Tasman Radiation Oncology Group trial 01.04. J. Clin. Oncol.,30: 3827-3833.

14. Arbea L, Ramos LI, Martínez-Monge R, Moreno $M$ and Aristu J (2010): Intensity-modulated radiation therapy (IMRT) vs. 3D conformal radiotherapy (3DCRT) in locally advanced rectal cancer (LARC): dosimetric comparison and clinical implications, Radiat.Oncolo., 5:7-17. 